\section{Atenção hospitalar perinatal e mortalidade neonatal no município de Juiz de Fora, Minas Gerais}

\section{Perinatal health care and neonatal mortality in the municipality of Juiz de Fora in the9* State of Minas Gerais}

Maria da Consolação Magalhães 1

Marilia Sá Carvalho 2

1 Secretaria Municipal de Sa de. Av. Bar o do Rio Branco, 2001/2307. Juiz de Fora, MG, Brasil.

CEP: 36.013-020

2 Escola Nacional de Sa de Publica. Funda o Oswaldo Cruz, Rio de Janeiro, RJ

\begin{abstract}
Objectives: to identify possible causes for the excessive rates of neonatal mortality in the municipality of Juiz de Fora and to assess the quality of hospital records.

Methods: a case control study based on information from the medical records of the three main maternity hospitals in the municipality. One hundred and three neonatal deaths were analyzed together with the sample of 232 liveborn babies.

Results: birth weight and Apgar index in the fifth minute were important predictive factors for neonatal deaths regardless of the maternity ward. The odd ratio in Hospital 1 was 3,97 times higher than in Hospital 3. Based on specialists' opinion, a medical record score was implemented which indicated that Hospital 1 had the lowest mean not only in relation to the cases but to the controls as well.

Conclusions: the absence of information adequately registered in medical records is an indicator of poor medical assistance and certainly, delays proper care. The investigation reported deficiencies, particularly in the records, of the perinatal care offered by the three maternity hospitals.
\end{abstract}

Key words Neonatal mortality, Services evaluation, Hospital records

\section{Resumo}

Objetivos: identificar os poss veis fatores que $t m$ contribu do para o excesso da mortalidade neonatal no munic pio de Juiz de Fora e avaliar a qualidade do preenchimento dos prontu rios hospitalares.

$M$ todos: estudo caso-controle baseado em informa es colhidas nos prontu rios das tr s principais maternidades do munic pio. Foram analisados 103 bitos neonatais e amostra de 232 nascidos vivos.

Resultados: as vari veis peso ao nascer e ndice de Apgar no quinto minuto foram importantes fatores preditivos para o bito neonatal, independente do local de nascimento. Quando se comparou, o risco de morrer, entre os hospitais verificou-se que no Hospital 1 o risco foi 3,97 vezes maior que no Hospital 3. Baseado em consulta a especialistas, foi criado um escore para avalia o do prontu rio, onde o Hospital 1 apresentou mediana mais baixa, tanto entre casos como em controles.

Conclus es: a aus ncia de informa es adequadamente registradas no prontu rio um indicador de precariedade na assist ncia, e, certamente, retarda a realiza o de conduta indicada. A pesquisa apontou defici ncias, particularmente nos registros, da assist ncia perinatal oferecida nos tr s hospitais. Palavras-chave Mortalidade neonatal, Avalia o dos servi os, Registros hospitalares 


\section{Introdução}

, medida que melhoram as condi es de vida e de acesso aos servi os b sicos de sa de, o perfil da mortalidade infantil se altera, passando a ter mais import ncia aspectos ligados aten o gesta o e ao parto, mortalidade neonatal. Esta compreende os bitos de crian as durante os primeiros 28 dias de vida, sendo subdividida em precoce, a que ocorre durante os primeiros sete dias de vida, e a tardia, entre o s timo e o vig simo oitavo dias de vida. A mortalidade perinatal aquela que ocorre entre 22 semanas completas de gesta o (154 dias ou 500 gramas) e termina com sete dias completos de vida. ${ }^{1}$

Os principais fatores de risco para a mortalidade neonatal e perinatal $\mathrm{s}$ o os ligados sa de da $\mathrm{m}$ e e qualidade da assist ncia gesta o. Entre os riscos associados sa de materna est o: idade materna, intervalo interpartal, altura, paridade, peso anterior e ganho de peso durante a gravidez, aborto, natimortos ou mortes neonatais pr vias e doen as maternas. Esses fatores podem causar hip xia neonatal, baixo peso ao nascer, prematuridade, $\mathrm{m}$-forma es con$\mathrm{g}$ nitas, entre outras. 2

$O$ peso ao nascer um indicador sens vel do estado de sa de de uma popula o e de avalia o dos programas de sa de e nutri o, principalmente do $\mathrm{n}$ vel local. 3 um dos principais determinantes da mortalidade neonatal. $O$ percentual de baixo peso no total de rec m-nascidos (RNs) de alguns pa ses no in cio da d cada de 80 foi: Estados Unidos 6,8\%, Chile 6,4\%, Costa Rica 6,6\%. Na cidade de Ribeir o Preto, SP, no per odo de 1968-1970, foi 8,7\%. Os fatores de risco mais comumente associados ao baixo peso ao nascer s o: $\mathrm{h}$ bito de fumar, hipertens o arterial cr nica, infec es perinatais cr nicas, desnutri o materna, entre outros. 4

A dura o da gesta o serve como indicador de risco para a mortalidade neonatal e est estreitamente relacionada com o baixo peso ao nascer. $\mathrm{S}$ o prematuros os nascimentos ocorridos antes de completadas 37 semanas. As principais causas s o: desnutri o, infec es, prematuridade iatrog nica, hipertens o, rotura prematura das membranas, entre outras. ${ }^{4}$ Entre os bitos neonatais ocorridos no Brasil, no per odo de 1990 a 1995, 30\% foram de crian as com menos de 37 semanas de gesta 0.2

Estudos apontam que o $\mathrm{n}$ mero de consultas realizadas durante o $\mathrm{pr}$-natal foi de muita import ncia para diminuir a gesta o de alto risco; quanto maior o $\mathrm{n}$ mero de consultas durante o pr -natal menor a mortalidade. Para que haja benef cios para $\mathrm{m}$ e e filho, o Minist rio da Sa de recomenda o m nimo de seis consultas de pr -natal. 5
Em estudo realizado em Pelotas, RS, comparando os anos de 1982 e 1993, observou-se que o coeficiente de mortalidade neonatal precoce sofreu uma redu o de 16,4/1.000 para 11,7/1.000 nascidos vivos. 6 A queda do coeficiente nos anos estudados foi mais acentuada nas faixas de renda at tr $\mathrm{s}$ sal rios $\mathrm{m}$ nimos e maior que 10 sal rios $\mathrm{m}$ nimos. Em 1993, o coeficiente de mortalidade neonatal precoce foi de 27,9,26,9/1.000 e 8,5/1.000 nascidos vivos para nenhuma consulta de pr -natal, uma a quatro consultas e mais de cinco consultas, respectivamente.

Outro fator que interfere na mortalidade perinatal a idade da m e: a mortalidade maior nos extremos da vida reprodutiva. 6

No Estado do Rio de Janeiro, Leal e Szwarcwald 7 estudaram a evolu o da mortalidade neonatal entre o per odo de 1979 e 1993 , verificando que a queda se deu de forma mais lenta do que a $\mathrm{p} \mathrm{s-}$ neonatal. No interior do estado o descenso foi menor que na capital. Os bitos ocorridos na primeira hora ap s o nascimento apresentaram aumento tanto na capital como no interior do estado, denunciando piora na qualidade da aten o ao parto.

Conforme os dados levantados do Sistema de Informa es sobre Mortalidade do Departamento de Epidemiologia da Secretaria Municipal de Sa de, a mortalidade infantil em Juiz de Fora vinha apresentando tend ncia de queda, com uma redu o de $57 \%$ no coeficiente entre 1980 e $1996(60,8 / 1.000$ e $26,2 / 1.000$, respectivamente). O mesmo acontecia com a mortalidade neonatal precoce, que sofreu uma redu o de $31 \%$, $(22,4 / 1.000$ e 17,1/1.000 nascidos vivos). Por m, observando os anos de 1995, 1996 e 1997, h um aumento na mortalidade neonatal precoce e neonatal tardia. Em $1998 \mathrm{j}$ se verifica um ligeiro decr scimo em rela o aos anos anteriores. ${ }^{8}$ A mortalidade neonatal precoce o componente que vem contribuindo para o aumento dos coeficientes de mortalidade infantil nesse per odo.

Os principais grupos de causas de mortalidade infantil no per odo de 1994 a 1997 foram, em primeiro lugar, as afec es originadas no per odo perinatal, (prematuridade, septicemia, an xia e hip xia, entre outras), totalizando 55\% em 1994 e $72,9 \%$ em 1997, seguidas pelas doen as do aparelho respirat rio ou pelas anomalias cong nitas. ${ }^{8}$

A mortalidade neonatal est associada qualidade da assist ncia prestada mulher durante o pr natal, parto e com os cuidados imediatos ao rec mnascido.

Embora existam $v$ rias defini es para qualidade da assist ncia $\mathrm{m}$ dica, ser adotada neste estudo a proposta de Donabedian, 9 em que a boa assist ncia 
deve proporcionar aos pacientes o $\mathrm{m}$ ximo poss vel de benef cios sem, no entanto, aumentar os riscos, utilizando para tal fim o conhecimento cient fico e tecnol gico dispon vel. A partir disso, Fernandez ${ }^{10}$ conceitua a qualidade do cuidado perinatal como sendo a aplica o de tecnologias obst tricas e neonatais, visando alcan ar os ndices mais baixos de mortalidade perinatal, utilizando-a portanto como o resultado do cuidado perinatal. Quando as novas tecnologias s o introduzidas sem a avalia o adequada de sua efetividade, os custos dos servi os tendem a crescer sem que necessariamente haja ganho para a sa de dos usu rios. ${ }^{10}$

O conceito de avalia o consiste em emiss o de julgamento, que se baseia numa ".. compara o entre aquilo que se deseja avaliar e uma situa o ideal ou equivalente"(Silva e Formigli; 1994: 84).11 Alguns trabalhos relacionados com a avalia o da qualidade da assist ncia perinatal revelaram a aten o ao $\mathrm{pr}$-natal de $\mathrm{m}$ dia ou baixa qualidade, 10 inadequa o da assist ncia ao parto, com altas taxas de ces reas, falta de registros adequados sobre o trabalho de parto, 12 bitos neonatais evit veis, 12,13 grau de implanta o do programa materno-infantil insatisfat rio, 14 e condi es sanit rias e de controle de infec o hospitalar em procedimentos de interesse para maternidades inadequados. 15 Steinwachs 16 afirma que a qualidade da assist ncia pode ser julgada como alta quando alguns elementos est o presentes: hist ria do paciente, achados no exame f sico que contribuem para o diagn stico e tratamentos espec ficos, e se esses achados mostram ser eficazes em modificar os resultados para o indiv duo. Os procedimentos escolhidos para esta avalia o s o aceitos na literatura e fazem parte das rotinas do Centro Latino Americano de Perinatologia (CLAP) da Organiza o Mundial da Sa de, ${ }^{17}$ para adequada assist ncia perinatal.

O principal objetivo deste estudo identificar os poss veis fatores que $\mathrm{t} \mathrm{m}$ contribu do para o excesso da mortalidade neonatal no munic pio de Juiz de Fora e avaliar a qualidade do preenchimento dos prontu rios hospitalares, contribuindo para reorientar o atendimento s parturientes e rec m-natos pelo Sistema nico de Sa de do munic pio.

\section{Métodos}

Foi realizado um estudo dos bitos neonatais, com desenho caso-controle, baseado em informa es colhidas em prontu rios dos tr s hospitais de Juiz de Fora. O munic pio de Juiz de Fora, MG, Brasil, tem aproximadamente 460.000 habitantes, 98\% dos quais em rea urbana, com cobertura de servi os urbanos - gua, esgoto, coleta de lixo e energia el trica - acima de $90 \%$ da popula o. A cidade tem sua volta uma rede de munic pios cujas popula es $\mathrm{v} \mathrm{m}$ em busca de assist ncia m dica, servi os educacionais e com rcio varejista. 18

No munic pio existem tr s hospitais-maternidade conveniados com o Sistema nico de Sa de (SUS), aqui denominados de Hospital 1, 2 e 3 . Os tr s oferecem est gios em obstetr cia atrav s de conv nio com a Universidade Federal de Juiz de Fora. O atendimento ao SUS realizado por plantonistas e residentes obstetras, pediatras e anestesistas, sendo que no Hospital 3 o anestesista fica em regime de sobreaviso (fora do hospital, por $\mathrm{m}$ acess vel por telefone) nos fins de semana. O alojamento conjunto parcial nos tr s hospitais: os $\mathrm{RN}$ recebem todos os cuidados no ber rio. Os profissionais $n$ o $t \mathrm{~m} v \mathrm{n}$ culo empregat cio com os hospitais, recebendo pagamento pelos procedimentos que realizam. Somente o Hospital 1 possui leitos de UTI neonatal (desde 1995), que servem ao SUS, num total de nove. O Hospital 3 um hospital geral, privado, possuindo 10 leitos de obstetr cia em conv nio com o SUS. Alguns obstetras plantonistas oferecem pr -natal no hospital, a crit rio de cada profissional. Esses hospitais atenderam cerca de $90 \%$ dos nascimentos registrados no munic pio de Juiz de Fora no per odo de julho de 1997 a dezembro de 1998. No per odo estudado ocorreram 3.470 nascimentos no Hospital 1, 2.608 no Hospital 2 e 3.314 no Hospital 3. Os coeficientes de mortalidade neonatal nos tr s Hospitais foram: 36,$9 ; 10,3$; e 17,8 por mil, para os hospitais 1,2 e 3 respectivamente. A mortalidade neonatal nos tr $\mathrm{s}$ hospitais, em rec m-nascidos com peso superior a 1.000 gramas, foi 26,$5 ; 8,5$ e 11,8 por 1.000 , para os hospitais 1,2 e 3 respectivamente.

A investiga o foi feita utilizando prontu rios hospitalares e informa es registradas em qualquer parte dos mesmos. Foram analisados todos os bitos de crian as menores de 28 dias, residentes no munic pio, levantados a partir do Sistema de Informa es de Mortalidade (SIM), ocorridos no munic pio de Juiz de Fora, no per odo de 1 de julho de 1997 a 31 de dezembro de 1998. Os controles foram obtidos de uma amostra de todos os nascimentos registrados no Sistema de Informa es de Nascidos Vivos (SINASC), no per odo de 1 de junho de 1997 a 31 de dezembro de 1998. A amostragem para composi o do grupo controle foi aleat ria simples, de todos os nascimentos com peso superior a 1.000 gramas. Os casos representam todo o universo da pesquisa e os controles uma amostra da popula o que produziu os casos. 
Foram exclu dos casos e controles com peso inferior a 1.000 gramas devido a diferen as do potencial de presta o de assist ncia m dica ao rec mnascido existente nos tr $\mathrm{s}$ hospitais, bem como os portadores de anomalias cong nitas, por necessitarem de servi os muito especializados. Tamb m exclu dos foram os partos gemelares, por terem probabilidades de sobreviv ncia mais baixas do que os nascimentos nicos. ${ }^{10}$ As perdas foram devidas $\mathrm{n}$ o localiza o dos prontu rios nos hospitais.

Um controle foi a bito depois de 28 dias, permanecendo no grupo controle. Um controle foi a bito antes de 28 dias, pertencendo, portanto, aos casos. Os casos em que na Declara o de bito (DO) n o constavam peso e tipo de gravidez foram inclu dos para estudo e descartados posteriormente, quando $\mathrm{n}$ o preenchiam os crit rios ap s verifica o no prontu rio.

A perda de prontu rios por n o localiza o nos arquivos hospitalares foi de $26,4 \%$ dos casos e $8,3 \%$ dos controles. Houve diferen a entre os hospitais ( $p$ $<0,001)$ e entre casos e controles no Hospital 1 e Hospital $3(\mathrm{p}<0,01)$.

A an lise dos dados foi feita considerando as informa es por hospital e por local de nascimento. Foi utilizado o Epi-info para c lculo do tamanho da amostra, digita o e an lise dos dados e SPSS para an lise. Foi calculada a raz o de chances para as vari veis que apresentavam acima de $15 \%$ de preenchimento.

Para avaliar a qualidade da assist ncia prestada a partir dos prontu rios se atribuiu a cada um, individualmente, um escore relacionado qualidade do registro. Baseado em consultas a especialistas neonatologistas, sobre a import ncia da informa o para identifica o dos fatores de risco para o $\mathrm{RN}$ e para o acompanhamento de estado de sa de da crian a, arbitrou-se um valor para cada vari vel, que oscilou entre 0 (zero) e 10 pontos. O valor zero foi atribu do aos campos $\mathrm{n}$ o preenchidos, e os valores cinco e 10 aos preenchidos. Cada prontu rio poderia somar de zero a 100 pontos, atribuindo-se o maior valor para o prontu rio com todas as informa es preenchidas e o menor valor para o prontu rio onde todas as informa es estavam ausentes. A pontua o foi da seguinte forma:

\section{Atenção pré-natal}

Grupo sang neo da $\mathrm{me}=5$ pontos; fator $\mathrm{RH}=5$ pontos; sorologia para $\mathrm{s}$ filis $=10$ pontos;

\section{Anamnese e exame físico}

Idade da $\mathrm{m} \mathrm{e}=5$ pontos; data da ltima menstrua $\mathrm{o}$ $=5$ pontos; apresenta o fetal $=5$ pontos;

dura o da gesta $\mathrm{o}=10$ pontos.

\section{Evolução do trabalho de parto}

Tempo de amniorrexe $=10$ pontos; monitoramento do batimento $\mathrm{c}$ rdio-fetal $=10$ pontos

\section{Condições de nascimento do RN}

Circular de cord o umbilical $=5$ pontos; presen a de mec nio $=10$ pontos; ndice de Apgar $=10$ pontos; peso ao nascer $=10$ pontos.

Devido ao grande $\mathrm{n}$ mero de aus ncia de informa es de algumas vari veis no prontu rio, as mesmas foram exclu das da an lise dos fatores de risco. As vari veis selecionadas para an lise de odds ratio (OR) foram: peso ao nascer, Apgar no primeiro e quinto minutos e monitoramento do batimento $\mathrm{c} r-$ dio-fetal (BCF).

\section{Resultados}

Ocorreram, nos tr s hospitais, 151 bitos de RNs cujo peso era superior a 1.000 gramas sendo selecionados 253 controles para estudo. Ao final, foram 103 casos e 232 controles estudados, sendo quatro bitos em nascimentos transferidos de hospitais $\mathrm{n} o$ participantes do estudo para o Hospital 1. Dos bitos ocorridos no Hospital 1, 12,9\% nasceram em outros hospitais.

O preenchimento dos prontu rios foi deficiente e com diferen as entre hospitais. Na Tabela 1 destacamse as vari veis que estavam ausentes em mais de $20 \%$ dos prontu rios. Entre as vari veis estudadas o maior ndice de $\mathrm{n}$ o registro das informa es foi verificado no Hospital 1. Devido precariedade dos registros, algumas vari veis no puderam ser utilizadas na an lise, tais como tempo de amniorrexe, apresenta o fetal, dura o da gesta o, crit rio para se conhecer a idade gestacional, entre outras. 
Percentual de omissão de informações sobre o pré-natal e parto, em casos e controles por hospital de nascimento. Juiz de Fora, MG, 1997 a 1998.

\begin{tabular}{|c|c|c|c|c|c|c|}
\hline \multirow[b]{2}{*}{ Informações } & \multicolumn{2}{|c|}{ Hospital 1} & \multicolumn{2}{|c|}{ Hospital 2} & \multicolumn{2}{|c|}{ Hospital 3} \\
\hline & $\begin{array}{l}\text { Casos } \\
(n=54)\end{array}$ & $\begin{array}{l}\text { Controles } \\
\qquad(n=70)\end{array}$ & $\begin{array}{l}\text { Casos } \\
(n=33)\end{array}$ & $\begin{array}{l}\text { Controles } \\
(n=76)\end{array}$ & $\begin{array}{l}\text { Casos } \\
(n=16)\end{array}$ & $\begin{array}{l}\text { Controles } \\
(n=86)\end{array}$ \\
\hline Duração da gestação & 13,0 & 42,8 & 30,3 & 30,3 & 0 & 9,3 \\
\hline Número de gestações & 13,0 & 4,3 & 3,0 & 1,3 & 0 & 1,2 \\
\hline $\begin{array}{l}\text { Monitoramento de batimento cárdio-fetal } \\
\text { (conforme o total de prontuários com o requesito) }\end{array}$ & 32,0 & 45,4 & 43,7 & 42,9 & 8,3 & 27,1 \\
\hline Presença de mecônio no líquido amniótico & 35,2 & 10,0 & 3,0 & 0 & 6,2 & 2,3 \\
\hline Tempo de amniorrexe & 37,0 & 47,1 & 3,0 & 7,9 & 12,5 & 18,6 \\
\hline Sorologia para sífilis & 59,0 & 81,4 & 90,9 & 92,1 & 87,5 & 79,1 \\
\hline Peso de nascimento & 0 & 0 & 15,2 & 1,3 & 6,2 & 3,5 \\
\hline Apresentação fetal & 70,4 & 53,5 & 9,1 & 2,6 & 0 & 2,3 \\
\hline Última menstruação & 92,6 & 92,8 & 36,4 & 26,3 & 38,5 & 27,9 \\
\hline Capurro & 79,6 & 90 & 86,1 & 84,2 & 93,7 & 95,3 \\
\hline
\end{tabular}

* Controle de batimento cárdio-fetal: quando o tempo entre a internação e o parto foi superior a duas horas.

A informa o sobre o hor rio de nascimento e do bito esteve ausente em $37,5 \%$ dos bitos ocorridos no Hospital 3. O Hospital 1 apresentou o menor percentual de bitos na primeira hora de vida $(1,6 \%)$, e o maior percentual a partir do terceiro dia de vida, sendo que $44 \%$ ocorreram no per odo neonatal tardio. Houve diferen a estatisticamente significativa $(p<0,001)$ entre a idade da crian a ao morrer no Hospital 1 e Hospital 2, sendo a idade mais alta para o primeiro hospital. A $m$ dia de idade da crian a ao morrer foi de 213 horas no Hospital 1, 69 horas no Hospital 2 e 73 horas no Hospital 3.

A mediana do $n$ mero de gesta es e de partos das m es do Hospital 2 foi superior aos demais, tanto entre casos como entre controles. Entre os casos n o houve diferen a estat stica entre o peso ao nascer no Hospital 1 e Hospital 2, havendo diferen a entre estes dois e o Hospital 3. Para os controles a diferen a entre os $\operatorname{tr} \mathrm{s}$ hospitais $\mathrm{n}$ o foi significativa. Cerca de 39\% dos casos pesavam entre 1.000 e 1.500 gramas. Foram a bito $27,9 \%$ de crian as com peso superior a 2.500 gramas. O Hospital 2 teve a maior propor o de casos e controles com peso menor que 2.500 gramas: 80,6 e $14,9 \%$, respectivamente

A raz o de chances (OR) de morrer ou n o, foi significativamente diferente de um entre os Hospi- 
tais 1 e $3(\mathrm{OR}=3,97)$ e entre os Hospitais 2 e $3(\mathrm{OR}$ $=2,4$ ) (Tabela 2).

Entre as vari veis analisadas (Tabela 3), o peso apresentou OR significativo e em torno de 21 para beb s com peso inferior a 2.500 gramas nos tr s hospitais; o ndice de Apgar entre zero a sete no primeiro minuto representou um risco de morrer substancialmente maior: 20,6, 28,3 e 53,7 vezes para os Hospitais 3,1 e 2 , respectivamente. No Hospital 3, o n o monitoramento do batimento $\mathrm{c}$ rdio-fetal foi fator de prote o $(\mathrm{OR}=0,11)$.
Tabela 2

Razão de chances de morrer entre os hospitais para casos e controles. Juiz de Fora, MG, 1997 a 1998.

\begin{tabular}{lcc}
\hline Hospitais & OR & IC95\% \\
\hline Hospital $1 \times 2$ & 1,66 & $0,94-2,94$ \\
Hospital $1 \times 3$ & 3,97 & $2,02-7,89$ \\
Hospital $2 \times 3$ & 2,40 & $1,18-4,89$ \\
\hline
\end{tabular}

Tabela 3

Distribuição e razão de chances de morrer de algumas variáveis dos casos e controles de três hospitais. Juiz de Fora, MG, 1997 a 1998.

\begin{tabular}{|c|c|c|c|c|c|c|}
\hline & Variável & Categoria & Casos & Controles & & \\
\hline \multirow{8}{*}{$\begin{array}{l}\frac{-}{\pi} \\
\frac{\pi}{0} \\
\frac{0}{0} \\
\frac{0}{1}\end{array}$} & Peso ao nascer & $<2.500$ & 40 & 8 & 20,7 & $(7,3-60,84)$ \\
\hline & & $\geq 2.500$ & 15 & 62 & & \\
\hline & Apgar primeiro minuto & $0-7$ & 33 & 5 & 28,3 & $(8,38-102,46)$ \\
\hline & & $8-10$ & 14 & 60 & & \\
\hline & Apgar quinto minuto & $0-7$ & 23 & 1 & 65,00 & $(8,32-1388,4)$ \\
\hline & & $8-10$ & 23 & 65 & & \\
\hline & Monitoramento batimento cardio-fetal & Não & 14 & 23 & 0,72 & $(0,3-1,7)$ \\
\hline & & Sim & 40 & 47 & & \\
\hline \multirow{8}{*}{$\begin{array}{l}\frac{N}{0} \\
\frac{\pi}{2} \\
\frac{2}{n} \\
\frac{0}{1}\end{array}$} & Peso ao nascer & $<2.500$ & 25 & 11 & 21,8 & $(6,59-77,0)$ \\
\hline & & $>2.500$ & 6 & 63 & & \\
\hline & Apgar primeiro minuto & $0-7$ & 26 & 4 & 53,7 & $(14,2-268,14)$ \\
\hline & & $8-10$ & 8 & 71 & & \\
\hline & Apgar quinto minuto & $0-7$ & 21 & 2 & 58,96 & $(11,01-421,94)$ \\
\hline & & $8-10$ & 13 & 73 & & \\
\hline & Monitoramento batimento cardio-fetal & Não & 21 & 36 & 1,67 & $(0,68-4,11)$ \\
\hline & & Sim & 14 & 40 & & \\
\hline \multirow{8}{*}{$\begin{array}{l}\frac{m}{\pi} \\
\frac{\pi}{2} \\
\frac{0}{n} \\
0 \\
\frac{0}{1}\end{array}$} & Peso ao nascer & $<2.500$ & 11 & 8 & 20,6 & $(4,87-94,54)$ \\
\hline & & $>2.500$ & 5 & 75 & & \\
\hline & Apgar primeiro minuto & $0-7$ & 11 & 8 & 20,6 & $(4,87-94,54)$ \\
\hline & & $8-10$ & 5 & 75 & & \\
\hline & Apgar quinto minuto & $0-7$ & 8 & 2 & 41,0 & $(6,20-349,33)$ \\
\hline & & $8-10$ & 8 & 82 & & \\
\hline & Monitoramento batimento cardio-fetal & Não & 1 & 33 & 0,11 & $(0,00-0,85)$ \\
\hline & & Sim & 15 & 53 & & \\
\hline
\end{tabular}

* Controle de batimento cárdio-fetal: quando o tempo entre a internação e o parto foi superior a duas horas. 
A mediana do escore do preenchimento foi mais baixa no Hospital 1, tanto nos casos como nos controles: 69,3 e 65,7 pontos. O hospital com o melhor resultado no preenchimento foi o Hospital 3 com a mediana de 86,8 e 84,5 pontos para casos e controles, respectivamente. No Hospital 2 a mediana do escore foi de 74,5 e 77,9 pontos para casos e controles, respectivamente (Figura 1).

\section{Figura 1}

Box plot do escore do preenchimento das informações dos prontuários em três hospitais, em casos e controles. Juiz de Fora, MG, 1997 a 1998.

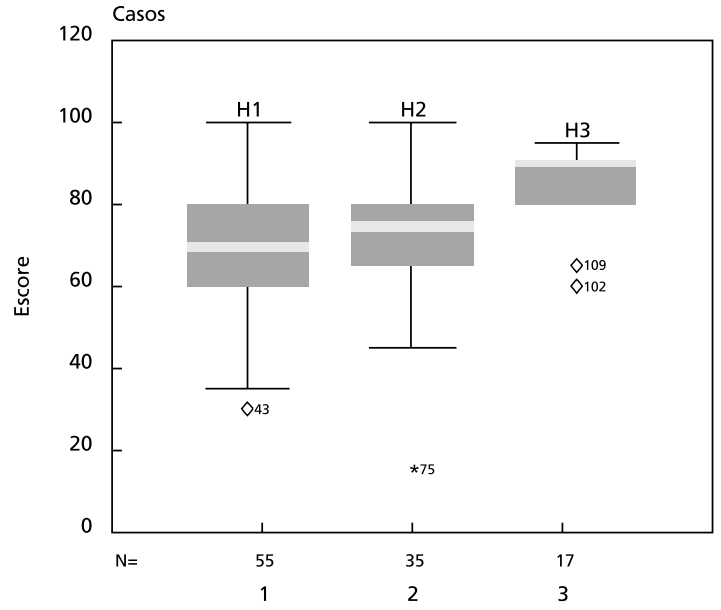

Local de nascimento

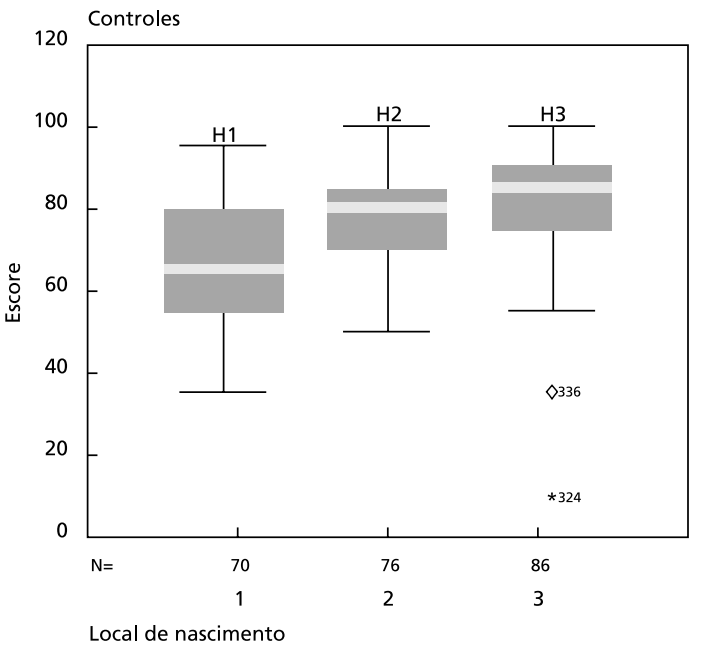

$\mathrm{H} 1=$ Hospital $1 ; \mathrm{H} 2=$ Hospital $2 ; \mathrm{H} 3=$ Hospital 3.

$\mathrm{Na}$ avalia o por prontu rio, verificou-se grande diferen a entre os hospitais. No Hospital 3 ocorreu o mais alto percentual de prontu rios com $80 \%$ ou mais das informa es presentes, sendo que os prontu rios dos casos foram mais preenchidos, com $82 \%$ acima de 80 pontos. No Hospital 1 observou-se o menor percentual de preenchimento das informa es, $\mathrm{n}$ o diferindo entre casos e controles, (cerca de 30\%).

\section{Discussão}

As diferen as nas perdas entre hospitais e entre casos e controles podem estar relacionadas com a gravidade dos casos. Entretanto, ainda que as perdas possam diminuir a validade dos resultados, a investiga o em servi os de sa de de grande import ncia para seu acompanhamento e avalia o. As fontes de informa o melhoram com o seu uso rotineiro. A perda dos casos, particularmente daqueles proposi- tadamente desaparecidos, apenas torna mais suave o quadro retratado, pelo aspecto quantitativo.

No Hospital 1 mais de $40 \%$ dos bitos ocorrem depois de sete dias de vida, havendo um adiamento da morte, padr o inverso do observado em outros trabalhos onde o maior percentual de bitos neonatais acontece nos primeiros dias de vida. 12,19 Leal e Swarcwald 7 em trabalho realizado no Rio de Janeiro, no per odo de 1979 a 1993, verificaram um aumento da mortalidade no primeiro dia, sendo que em 1993, 24,6\% dos bitos ocorreram em crian as com menos de 24 horas de vida, indicando uma piora na qualidade da assist ncia ao parto e neonato. A sobreviv ncia dos RNs nos primeiros dias, morrendo tardiamente, pode ser resultado de boas condi es de assist ncia imediata ao nascimento, mas que $\mathrm{n}$ o se traduz em sobreviv ncia, seja pelas condi es em que se deram os nascimentos ou por defici ncias na condu o do tratamento. Uma boa assist ncia ao RN ap s o parto e aparatos tecnol gicos nem sempre s o 
suficientes para reparar um pr-natal ou pr tica obst trica de $m$ qualidade.

Os coeficientes de mortalidade neonatal por hospital revelam uma alta mortalidade no Hospital 1. Pelos dados secund rios, se supunha que os RNs estariam falecendo tardiamente, depois de sete dias de vida, porque ao receberem alta, as condi es adversas em que viviam as fam lias $\mathrm{n}$ o asseguravam a sobreviv ncia de RNs de risco e eles retornavam ao hospital, onde ocorria o bito. No entanto, verificouse que os RNs n o recebem alta antes do bito e os bitos se deram em fun o de causas ligadas ao parto e p s-parto ou relacionadas aos cuidados recebidos.

O risco de morrer, quando relacionado com o peso ao nascer entre os hospitais foi alto em todos os hospitais, confirmando o baixo peso como principal determinante da mortalidade neonatal.2,3,6,10,19

O baixo peso demonstrou ser importante fator de risco para o bito independente do local de nascimento, demonstrando que as condi es em que se deram a gesta o e o acompanhamento da mesma se refletem na mortalidade neonatal. Entretanto, o grande $\mathrm{n}$ mero de bitos de crian as de peso adequado e idade gestacional a termo reflete defici ncias na qualidade da assist ncia pr -natal ou parto, ou ambas, nos tr s hospitais. O Apgar no quinto minuto foi outro fator de risco para o $\mathrm{RN}$ em todos os hospitais, indicando a import ncia da vigil ncia adequada do trabalho de parto e parto e assist ncia adequada logo ap s o nascimento.

Considerando que os profissionais trabalham em regime de plant o e que as usu rias do SUS s o atendidas por plantonistas, que se revezam a cada $12 \mathrm{ou}$ 24 horas, $n$ o estando garantido assim o atendimento pelo mesmo profissional, a aus ncia de informa es no prontu rio pode dificultar o acompanhamento e a avalia o do trabalho de parto e do RN, bem como a detec o precoce de intercorr ncias que possam afetar $\mathrm{m}$ e e filho. A qualidade do preenchimento dos dados nos prontu rios hospitalares estudados no difere dos trabalhos $\mathrm{j}$ publicados sobre o assunto, onde os registros s o bastante deficit rios. Em trabalho realizado no Rio de Janeiro em 1986 a 1987, $25 \%$ da amostra dos prontu rios n o apresentavam o registro de Apgar no primeiro minuto e 51\% no quinto minuto. $12 \mathrm{Os}$ tr $\mathrm{s}$ hospitais investigados recebem estagi rios do curso de medicina e portanto se esperaria uma melhor qualidade dos registros, visto que os alunos est o em fase de aprendizagem. Uma boa anamnese, um adequado acompanhamento do trabalho de parto, com boa qualidade dos registros, seria o $\mathrm{m}$ nimo esperado em um hospital com atividade de ensino.

O descaso com os registros pode estar comprometendo a qualidade da assist ncia prestada. Em todos os hospitais verificou-se baixa qualidade dos registros em prontu rios. Os resultados indicam $m$ qualidade de assist ncia, inclusive no Hospital 1, hospital que oferece servi os e tecnologia de alta complexidade.

importante que se incorpore a avalia o da assist ncia perinatal na rotina do SUS e n o somente ao atender demandas pontuais por auditoria, atrav $\mathrm{s}$ de um servi o de avalia o permanente para os hospitais conveniados e a rede b sica de sa de. Neste contexto, a cria o de mecanismos de incentivo para os servi os com resultados favor veis $m$ e e ao $\mathrm{RN}$, elevaria a qualidade da assist ncia. fundamental tamb m que sejam implantados comit s de estudos sobre a mortalidade perinatal, com o objetivo de avaliar permanentemente todos os bitos, visando detectar as falhas e propor rotinas de atendimento parturiente e ao $\mathrm{RN}$, tais como protocolos de atendimento e de controle de infec es.

\section{Referências}

1. OMS (Organiza o Mundial da Sa de). Classifica o estat stica internacional de doen as e problemas relacionados a sa de (CID). 10. rev. S o Paulo: Edusp; 1994.

2. Maranh o AGK, Joaquim MMC, Siu C. Mortalidade perinatal e neonatal no Brasil. Tema Radis 1999; 17: 6-16.

3. Puffer RR, Serrano CV. Caracter sticas del peso al nacer. Washington (DC): OPS; 1988. (Publicaci n Cientifica, 504).
4. Minist rio da Sa de. Secretaria de Assist ncia Sa de. Coordena o Materno-Infantil. Manual de assist ncia ao rec m-nascido. Bras lia (DF): O Minist rio; 1994.

5. Minist rio da Sa de. INAN (Instituto Nacional de Alimenta o e Nutri o). Assist ncia integral sa de da mulher: base para uma a o program tica. Bras lia (DF): O Minist rio; 1984 
6. Menezes AMB, Barros FC, Victora CG, Alves C, Rocha C, Albernaz E, Menezes FS, Janke H. Mortalidade perinatal em duas coortes de base populacional no Sul do Brasil: tend ncias e diferenciais. Cad Sa de P blica 1996; 12 Supl 1: 33-41.

7. Leal MC, Szwarcwald CL. Evolu o da mortalidade neonatal no estado do Rio de Janeiro, Brasil (1979-1993): an lise por causa segundo grupo de idade e regi o de resid ncia. Cad Sa de P blica 1996; 12: 243-52.

8. Magalh es MC. Aten o hospitalar perinatal e a mortalidade neonatal no munic pio de Juiz de Fora, MG [disserta o mestrado]. Rio de Janeiro: Escola Nacional de Sa de P blica da Funda o Oswaldo Cruz; 2000.

9. Donabediam A. La calidad de la atenci n medica: definici n e $m$ todo de evaluaci n. Mexico: La Prensa M dica; 1984.

10. Fern ndez JLB. Quality of perinatal medical care in Mexico city: an epidemiological study on the effects of medical care quality on perinatal mortality. M xico: La Prensa M dica; 1984

11. Silva LMV, Formigli VLA. Avalia o em sa de: limites e perspectivas. Cad Sa de P blica 1994; 10: 80-91.

12. Carvalho ML. Mortalidade neonatal e aspectos da qualidade da aten o sa de na Regi o Metropolitana do Rio de Janeiro em 1986/87 [disserta o mestrado]. Rio de Janeiro: Escola Nacional de Sa de P blica da Funda o Oswaldo Cruz; 1993.

13. Carvalho DS. Avalia o da qualidade de pr -natal, parto e puerp rio em Curitiba: uma an lise preliminar. In: $\mathrm{Cu}-$ ritiba. Secretaria de Sa de. Valorizando a dignidade materna. Curitiba: A Secretaria; 1996. p. 19-48.

14. Hartz ZM, Champagne F, Contandriopoulos A, Leal MC. Avalia o do programa materno-infantil: an lise de implanta o em sistemas locais de sa de no Nordeste do Brasil. In: Hartz ZMA. Avalia o em sa de, dos modelos conceituais pr tica na an lise da implanta o de programas. Rio de Janeiro: FIOCRUZ; 1997. p. 89-131.

15. Kishida GSN, Manzochi AMP, Cruz E, Bencke L. Alguns aspectos da quest o das maternidades de Curitiba: condi o sanit ria e de controle de infec o. In: Curitiba. Secretaria de Sa de. Valorizando a dignidade materna. Curitiba: A Secretaria; 1996. p. 55-68.

16. Steinwachs DM. Management information in decisionmaking. In: Armenian HK, Shapiro S. Epidemiology and health services. New York: Oxford University; 1998. p. 41-59.

17. OMS (Organiza o Mundial da Sa de), CLAP (Centro Latino Americano de Perinatologia). Sa de reprodutiva materna e perinatal: aten o pr -natal e do parto de baixo risco. Montivid u: A Organiza o; 1996. (Publica o Cient fica, 1321.03).

18. Juiz de Fora. Secretaria de Sa de. Plano municipal de sa de. Juiz de Fora: A Secretaria; 1997.

19. Coutinho SB. Mortalidade neonatal em cinco maternidades da cidade do Recife, 1994: relat rio final de pesquisa apresentado ao UNICEF. Recife: UNICEF; 1996.

Recebido em 29 de novembro de 2002

Vers o final reapresentada em 20 de maio de 2003

Aprovado em 10 de junho de 2003 